\title{
Asymptomatic Testicular Adrenal Rest Tumours in Adolescent and Adult Males with Congenital Adrenal Hyperplasia: Basal and Follow-up Investigation After 2.6 Years
}

\author{
Nike 'M.M.L. Stikkelbroeck' ${ }^{1}$, Ad R.M.M. Hermus ${ }^{2}$, Harold M. Suliman ${ }^{3}$, Gerrit J. Jager ${ }^{3}$ and Barto J. Otten ${ }^{1}$ \\ Departments of ${ }^{I}$ Paediatric Endocrinology, ${ }^{2}$ Endocrinology and ${ }^{3}$ Radiology, \\ University Medical Centre Nijmegen, Nijmegen, The Netherlands
}

\begin{abstract}
Aim: To study the course of asymptomatic testicular adrenal rest tumours in patients with congenital adrenal hyperplasia (CAH) and the association between tumour changes and glucocorticoid therapy adjustments.

Patients and Methods: Fifteen male patients with CAH (21-hydroxylase deficiency), in whom asymptomatic testicular adrenal rest tumours had been found at a baseline investigation, underwent scrotal ultrasonography and venous blood sampling (for LH, FSH and testosterone) on average 2.6 years later. The level of hormonal control was assessed by measurement of androstenedione in three diurnal saliva samples. Data on changes in glucocorticoid therapy since baseline were obtained from the patients' records.

Results: Tumour decrease, defined as $\geq 30 \%$ decrease in the sum of the longest diameter(s) of the lesion(s), was found in six patients; tumour increase, defined as $\geq 20 \%$ increase, in six and stable tumours in three patients. All three patients with overtreatment showed tumour decrease and of the six patients with undertreatment only one showed tumour decrease. Tumour increase was not only observed in undertreated patients but also in patients with adequate treatment. Changing the night dose of hydrocortisone into dexamethasone, to obtain
\end{abstract}

Reprint address:

Dr. B.J. Otten, M.D., Ph.D.

435 Paediatric Endocrinology

University Medical Centre Nijmegen

PO Box 9101

6500 HB Nijmegen, The Netherlands

e-mail: b.otten@cukz.umcn.nl prolonged ACTH suppression, had resulted in better adrenal suppression in only one patient. Conclusions: Tumour decrease could be achieved by aiming at adrenal oversuppression, but the required high glucocorticoid doses may induce side effects. In asymptomatic tumours in young male patients with $\mathrm{CAH}$, a practical guideline could be to optimise adrenal suppression to a maximal tolerable glucocorticoid dose and to offer analysis and cryopreservation of semen as soon as the patient can be motivated.

\section{KEY WORDS}

congenital adrenal hyperplasia, testicular adrenal rest tumours, glucocorticoids

\section{INTRODUCTION}

Congenital adrenal hyperplasia (CAH) is an inherited disorder of adrenal steroidogenesis, usually caused by 21 -hydroxylase deficiency. Cortisol (and aldosterone) production is decreased or absent, resulting in increased pituitary secretion of corticotropin (ACTH) and, consequently, excess adrenal androgen production. Treatment consists of administration of glucocorticoids (and mineralocorticoids) for cortisol (and aldosterone) substitution and suppression of excess androgen production by re-establishing the negative feedback on ACTH secretion ${ }^{1}$.

In male patients with $\mathrm{CAH}$, testicular adrenal rest tumours are increasingly reported at all ages ${ }^{2-4}$. The tumours are considered to be aberrant adrenal tissue that has descended with the testes and has become hyperplastic due to ACTH stimulation. The recommended treatment consists of increasing the 
glucocorticoid dose to suppress ACTH secretion ${ }^{5}$. Most reports on treatment describe patients who experienced testicular pain or subfertility. In these symptomatic cases increasing the dose of glucocorticoids is justified and often successful ${ }^{6,7}$. In asymptomatic cases, however, this approach is not self-evident because the relevance of the tumours is unknown and treatment with high glucocorticoid doses for a prolonged time induces side effects, such as weight gain, striae and osteoporosis. In this situation, the negative effects of increasing the glucocorticoid dose may exceed the benefits.

In a previous study, we have reported a high prevalence of testicular adrenal rest tumours in postpubertal male patients with $\mathrm{CAH}^{4}$. The tumours were all asymptomatic and the patients did not have immediate fertility interest. To study the course of asymptomatic testicular adrenal rest tumours, we re-evaluated the tumours on average 2.6 years after the baseline investigation. In addition, we studied the association between tumour changes and glucocorticoid therapy adjustments in the follow-up period.

\section{PATIENTS AND METHODS}

\section{Patients}

At the baseline investigation, all male patients who were seen in our centre for treatment of $\mathrm{CAH}$ due to 21-hydroxylase deficiency and who were 16 years or older, had participated $(n=17)^{4}$. In 16 of them, testicular adrenal rest tumours had been found. At the follow-up investigation, 15 of these 16 patients were included; the other patient did not want to have scrotal ultrasonography again. Patient characteristics have been extensively described elsewhere $e^{4}$ and, for comparison, the same patient numbers are used. In 12 of the 15 patients, saltwasting $\mathrm{CAH}$ had been diagnosed in the first year of life; three other patients had presented with simple virilizing $\mathrm{CAH}$ at the age of 2.9 years (one patient) and 6.2 years (two patients). In all 15 patients the diagnosis had been confirmed by DNA analysis. The patients were treated from the time of CAH diagnosis with glucocorticoids (and mineralocorticoids) and were followed up regularly by a(n) (paediatric) endocrinologist.

\section{Baseline investigation}

The baseline investigation in 1998 was crosssectional and consisted of scrotal ultrasonography, determination of plasma testosterone, serum $\mathrm{LH}$ and FSH, salivary 17-hydroxyprogesterone and androstenedione and semen analysis ${ }^{4}$.

\section{Period between baseline and follow-up investigation}

Routine clinic visits (2-4 times a year) consisted of history taking, physical examination (weight, blood pressure, Cushing signs) and determination of 17-hydroxyprogesterone and androstenedione in diurnal saliva samples. Analysis and cryopreservation of semen was offered to the patients. Semen analysis was performed according to WHO guidelines ${ }^{8}$.

Data on glucocorticoid therapy between baseline and follow-up investigation were retrospectively obtained from the patient records. To calculate the total glucocorticoid dose, doses of dexamethasone were converted into hydrocortisone equivalents ( 30 $\mathrm{mg}$ hydrocortisone $=0.75 \mathrm{mg}$ dexamethasone $)^{9}$.

\section{Follow-up investigation}

The follow-up investigation in 2001, $2.6 \pm 0.2$ years (mean $\pm \mathrm{SD}$ ) after the baseline investigation, consisted of scrotal ultrasonography, venous blood sampling and saliva sampling. Grayscale and colour Doppler ultrasonography were performed as at the baseline investigation ${ }^{4}$, by the same staff radiologist (GJJ), who was not informed about the therapeutic regimens. Ultrasonography results at follow up were compared to those at baseline: tumour decrease was defined as $\geq 30 \%$ decrease in the sum of the longest diameter(s) of the lesion(s), tumour increase was defined as $\geq 20 \%$ increase in the sum and the remaining category was called stable tumour. These criteria were derived from the RECIST (Response Evaluation Criteria in Solid Tumours) criteria ${ }^{10}$.

Venous blood sampling was performed at 09.00 $\mathrm{h}$ to measure levels of testosterone, $\mathrm{LH}$ and FSH. Patients had taken their regular morning medication. Plasma testosterone was measured by RIA after a paper chromatographic purification step ${ }^{11}$; the normal range in adult males is $11-45 \mathrm{nmol} / 1$. 
Serum LH and FSH were quantitatively determined (AxSYM, Abbott Laboratories, USA); the normal range in adult males is $1.4-8.5 \mathrm{U} / 1$ for $\mathrm{LH}$ and 1.5-11 U/l for FSH.

\section{Levels of salivary 17-hydroxyprogesterone and androstenedione}

Measurement of salivary levels of 17-hydroxyprogesterone and androstenedione is routinely used to assess hormonal control in patients with $\mathrm{CAH}^{12}$. Patients collected three saliva samples, by salivation into plastic cups, 2 days before the follow-up investigation, at $08.00 \mathrm{~h}, 12.00 \mathrm{~h}$, and $20.00 \mathrm{~h}$, before every regular glucocorticoid intake. In the samples, 17-hydroxyprogesterone and androstenedione levels were determined by RIA after a paper chromatographic purification step $^{13}$. The mean androstenedione level of the three samples was calculated to assess hormonal control: undertreatment was defined as a mean level above the reference morning $(08.00 \mathrm{~h})$ range $(>0.63 \mathrm{nmol} / 1)$, overtreatment below this range $(<0.14 \mathrm{nmol} / 1)$, and adequate treatment within the reference range, i.e. $0.14-0.63 \mathrm{nmol} / 1^{4}$.

\section{RESULTS}

\section{Results of the follow-up investigation}

Testicular tumours had increased in six patients $(6 / 15,40 \%)$. In one of these patients (\#8), the initial tumours in one testis had disappeared, and in the contralateral testis, tumours had appeared. Tumours had decreased in six patients $(6 / 15,40 \%)$, and were stable in three patients $(3 / 15,20 \%)$ (Table 1$)$.

Plasma testosterone levels were below the normal range in four of the 15 patients (Table 2). Three patients had decreased levels of both serum LH and FSH (hypogonadotropism); in two of them $(\# 3,4)$ this had been found also at baseline investigation; in the third patient serum LH had been normal and serum FSH had been elevated at baseline (\#6).

Overtreatment was found in three patients, undertreatment in six patients and adequate treatment in six patients (Table 2). The patients who were judged to be overtreated at the baseline investigation were still overtreated or adequately treated $(\# 7,9,13)$. The patients who were undertreated at baseline were still undertreated $(\# 3,4,10$, $17)$, except for one patient who was now overtreated (\#2).

Glucocorticoid therapy between baseline and followup investigation

Data on glucocorticoid therapy are shown in Table 3. In five patients $(\# 3,9,10,11,13)$, the glucocorticoid dose had not changed during the follow-up period and in two patients $(\# 4,7)$ the glucocorticoid dose had been diminished compared to the baseline situation. In one patient (\#2), the regimen of hydrocortisone 2 times a day $(15+10$ $\mathrm{mg}$ ) was changed into dexamethasone 3 times a day $(0.25+0.25+0.25 \mathrm{mg})$, to improve semen quality after oligoasthenoteratozoospermia was found and he and his wife wanted to have a child. In seven patients $(\# 5,6,8,12,15,16,17)$, the hydrocortisone regimen ( 3 times a day) had been changed into hydrocortisone in the morning (and afternoon) and dexamethasone in the evening. Changes in body mass index between baseline and follow-up investigation did not show a significant correlation with changes in glucocorticoid dose (Spearman rank, $\mathrm{p}>0.2$ ).

Relationship between tumour change, suppression of salivary androstenedione and glucocorticoid therapy

Tumour decrease was found in all three patients who were overtreated at the follow-up investigation; they were at baseline overtreated $(n=1)$, adequately treated $(n=1)$ or undertreated $(n=1)$. Tumour decrease was also found in two patients who were adequately treated at the follow-up investigation and overtreated at baseline, and in one patient who was still, although to a lesser degree, undertreated (\#17).

Tumour increase was found in three patients who were undertreated at the follow-up investigation and who were at baseline undertreated $(n=1)$ or adequately treated $(n=2)$. Tumour increase was also found in three patients who were adequately treated on both occasions.

Stable tumours were found in one patient with one small tumour who was adequately treated on both occasions (\#11) and in two patients with large 


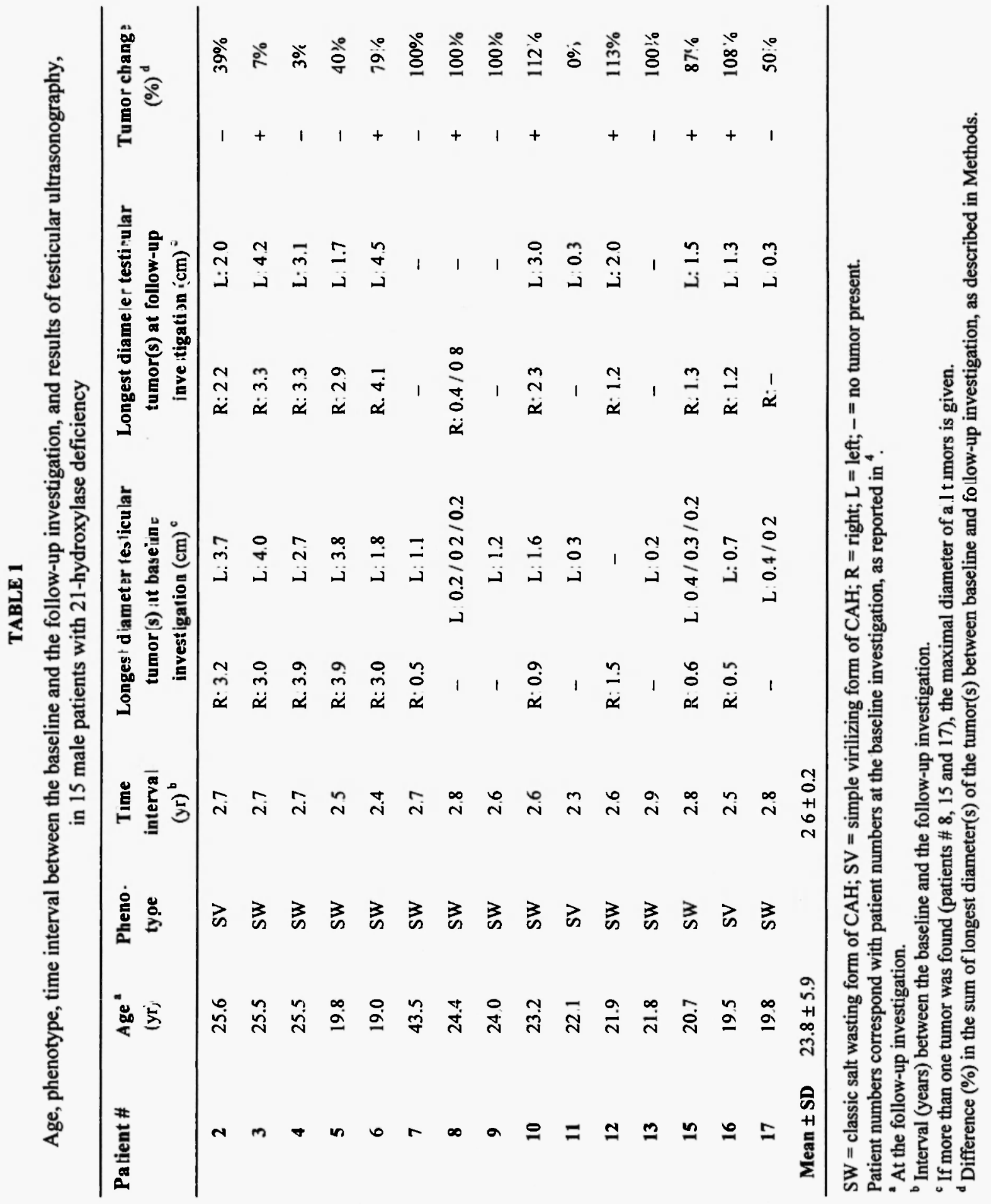

JOURNAL, OI: PIIDIA IRIC IENDOCRINOI,OGY \& METABOLISM 
TABLE 2

Plasma levels of testosterone, serum levels of LH and FSH, mean salivary levels of 17-hydroxyprogesterone (17-OHP) and androstenedione (adione) in 15 male patients with 21 -hydroxylase deficiency

\begin{tabular}{|c|c|c|c|c|c|c|c|}
\hline \multirow{2}{*}{$\begin{array}{c}\text { Patient \# } \\
2\end{array}$} & \multirow{2}{*}{$\begin{array}{c}\begin{array}{c}\text { Plasma } \\
\text { testosterone } \\
(\mathrm{nmol} / \mathrm{l})\end{array} \\
7.0\end{array}$} & \multirow{2}{*}{$\begin{array}{c}\begin{array}{c}\text { Serum LH } \\
(\mathrm{U} / \mathrm{l})\end{array} \\
2.5\end{array}$} & \multirow{2}{*}{$\begin{array}{c}\begin{array}{c}\text { Serum FSH } \\
(\mathrm{U} / \mathrm{l})\end{array} \\
11.1\end{array}$} & \multicolumn{2}{|c|}{$\begin{array}{c}\text { Mean salivary } \\
\text { 17-OHP } \\
(\mathrm{nmol} / \mathrm{l})^{\mathrm{a}} \\
\end{array}$} & \multicolumn{2}{|c|}{$\begin{array}{c}\text { Mean salivary } \\
\text { adione } \\
(\mathrm{nmol} / \mathrm{l})^{\mathrm{a}} \\
\end{array}$} \\
\hline & & & & 3.90 & 0.03 & 1.80 & 0.08 \\
\hline 3 & 27.0 & $<0.2$ & $<0.2$ & 6.24 & 7.26 & 2.93 & 4.80 \\
\hline 4 & 9.4 & 0.2 & 0.6 & 8.30 & 9.37 & 4.23 & 5.87 \\
\hline 5 & 12.0 & 5.8 & 9.4 & 0.83 & 0.13 & 0.22 & 0.09 \\
\hline 6 & 22.5 & 0.3 & 1.4 & 1.29 & 14.5 & 0.22 & 3.33 \\
\hline 7 & 16.0 & 3.8 & 14.6 & 0.02 & 0.05 & 0.12 & 0.10 \\
\hline 8 & 26.0 & 5.0 & 5.0 & 0.95 & 5.13 & 0.37 & 0.97 \\
\hline 9 & 12.0 & 2.9 & 1.9 & 0.01 & 1.73 & 0.06 & 0.45 \\
\hline 10 & 7.0 & 0.5 & 1.6 & 5.60 & 5.91 & 1.40 & 1.52 \\
\hline 11 & 9.0 & 9.6 & 16.7 & 1.19 & 1.30 & 0.19 & 0.16 \\
\hline 12 & 15.0 & 2.3 & 2.7 & 0.43 & 0.17 & 0.30 & 0.14 \\
\hline 13 & 21.0 & 8.6 & 10.8 & 0.06 & 0.38 & 0.10 & 0.17 \\
\hline 15 & 19.0 & 2.6 & 7.9 & 0.09 & 0.08 & 0.20 & 0.21 \\
\hline 16 & 27.0 & 4.4 & 6.7 & 0.76 & 0.19 & 0.25 & 0.18 \\
\hline 17 & 17.0 & 1.0 & 4.5 & 4.90 & 2.73 & 1.05 & 0.66 \\
\hline Normal values & $11.0-45.0$ & $1.4-8.5$ & $1.5-11.0$ & \multicolumn{2}{|c|}{$0.05-0.36^{b}$} & \multicolumn{2}{|c|}{$0.14-0.63^{b}$} \\
\hline
\end{tabular}

Numbers in italics are the levels at the baseline investigation.

"Salivary levels of 17-OHP and adione are mean levels from 3 samples (see Methods).

${ }^{b}$ Normal morning $(08.00 \mathrm{~h}$ ) levels; undertreatment was defined as a mean salivary adione level $>0.63 \mathrm{nmol} / \mathrm{h}$, overtreatment $<0.14 \mathrm{nmol} / 1$, adequate treatment $0.14-0.63 \mathrm{nmol} / 1$.

bilateral tumours $(\# 3,4)$ who were undertreated on both occasions.

The nine patients who at the time of the followup investigation had hydrocortisone in the morning (and afternoon) and dexamethasone at night, showed at the follow-up investigation overtreatment $(n=1)$, adequate treatment $(n=5)$ or undertreatment $(n=3)$.

The patient who was treated with dexamethasone $0.75 \mathrm{mg}$ daily to improve fertility (\#2), showed overtreatment at the follow-up investigation (undertreatment at baseline), decreased tumour size and improved semen quality. Spontaneous conception occurred a few weeks after the followup investigation and a healthy daughter has been born. Confirmation of parenthood was not performed.

\section{DISCUSSION}

The aim of this investigation was to study the course of asymptomatic testicular adrenal rest tumours in male patients with $\mathrm{CAH}$, and the association between tumour change and gluco- 


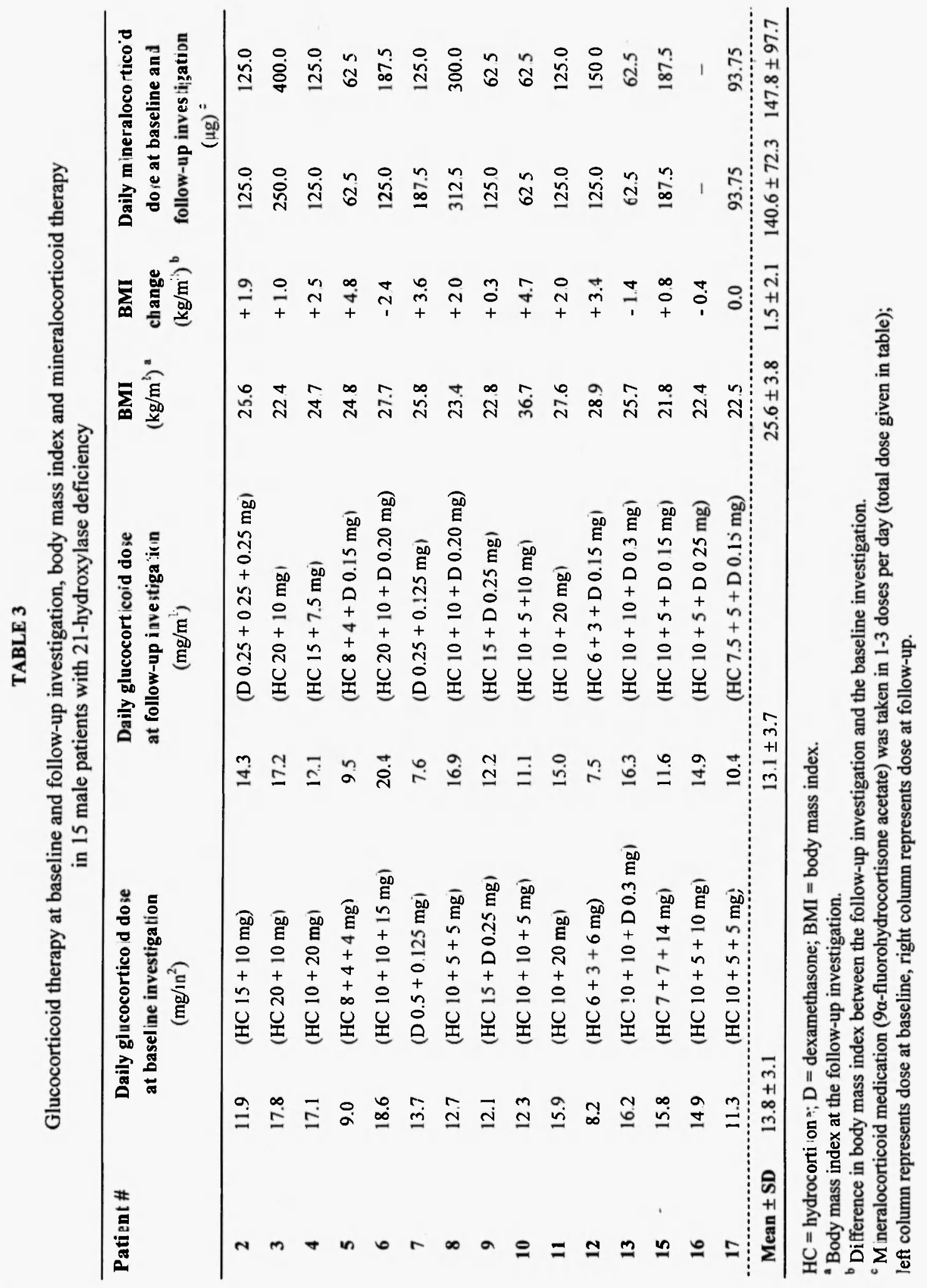

IOURNAL OI: PEDIATRIC ENDOCRINOLOGY \& METABOLISM 
corticoid therapy adjustments. This follow-up investigation, 2.6 years after a baseline investigation, showed tumour decrease in $40 \%$ of the patients and increase in $40 \%$. There was an association between tumour change and hormonal control: all three patients with overtreatment at follow-up showed tumour decrease and of the six patients with undertreatment only one showed tumour decrease. Tumour increase, on the other hand, was not only observed in undertreated patients but also in patients who were adequately treated at follow-up. These results suggest, like previous reports on symptomatic tumours, that tumour shrinkage can be achieved effectively by aiming at overtreatment. If the tumour does not shrink despite overtreatment or if there is persistent azoospermia despite tumour shrinkage, surgical intervention should be considered ${ }^{7}$.

In one patient, who had bilateral testicular tumours and oligoasthenoteratozoospermia, the glucocorticoid dose had been increased as recommended in symptomatic tumours ${ }^{5}$, because he and his wife wished to have a child. The tumour decrease and improvement of semen quality correspond with previous reports on successful treatment of symptomatic tumours in males with $\mathrm{CAH}^{6,7}$. Despite successful conception, testicular function was still not normal, based on the slightly increased serum FSH level and the decreased plasma testosterone level.

In the other 14 patients, our approach was aimed at tumour decrease by optimising adrenal suppression with glucocorticoid therapy adjustments, but avoiding additional glucocorticoid side effects such as weight gain and striae. In the patients with a hydrocortisone regimen (2-3 times a day) at baseline, replacing the night dose of hydrocortisone by dexamethasone was considered. Dexamethasone has a longer half life compared to hydrocortisone and it could, when given in the evening, lead to a longer suppression of the hypothalamo-pituitaryadrenal axis during the night ${ }^{14}$. However, this change of the glucocorticoid regimen resulted in better hormonal control in only one patient. In two patients there was even worse suppression: adequate treatment at baseline and undertreatment at follow-up, despite obviously increased glucocorticoid dose. This paradoxical result could suggest poor compliance or secretion of androstenedione by the testicular tumour itself. In that case, the androstenedione level is not only the result of adrenal activity but also of tumour activity and cannot be used to assess adrenal suppression ${ }^{15,16}$.

The adrenal rest tumours may not only be sensitive to hormonal control but also to other factors. This is illustrated by two cases of tumour regression despite worse adrenal suppression (overtreatment at baseline, adequate treatment at follow up) and the observation in another patient that unilateral tumours had disappeared and new tumours had appeared in the contralateral testes. Interestingly, Benvenga et al. suggested, from observations in a young male patient with $\mathrm{CAH}$, that testicular adrenal rest tumours may not only be sensitive to ACTH but also to $\mathrm{LH}^{17}$.

In three patients, hypogonadotropism was found and they were all undertreated. This corresponds to the hypothesis that hypogonadotropism in males with $\mathrm{CAH}$ is caused by suppression of the hypothalamo-pituitary-gonadal axis by adrenal androgens, both directly and after conversion to estrogens $^{18}$. Thus, in undertreated patients, fertility may not only be disturbed by testicular tumours but also by secondary hypogonadotropism.

This study provides data about the course of asymptomatic testicular adrenal rest tumours in males with $\mathrm{CAH}$ and the association with hormonal control, but there are some limitations. The adjustments in the glucocorticoid therapy during the follow-up period were based on the general principle of optimising adrenal suppression, but were made in every patient individually. There was no randomisation or standardised intervention, which makes the retrospective assessment of the relationship between tumour change and intervention difficult. Furthermore, the judgement of hormonal control was based on salivary androstenedione levels on only one day, which does not necessarily correspond with the hormonal control during the complete follow-up period. Finally, the outcome measure tumour change is only a surrogate for the major interest, which is future fertility. We considered, however, that tumour growth is an important measure because it will finally affect fertility by causing compression of residual testicular tissue and obstruction of semen flow. 
In conclusion, treating asymptomatic testicular adrenal rest tumours in young patients with $\mathrm{CAH}$ is difficult because the natural course of the tumours and their impact on fertility are unknown, the patient may not yet be interested in fertility, and, furthermore, treatment with increased doses of glucocorticoids can induce side effects. The tumours should be detected as early as possible, and therefore we would recommend performing testicular ultrasonography in all male patients with $\mathrm{CAH}$ on a regular basis (e.g. every 1-2 years) from the onset of puberty (testicular volume of $4 \mathrm{ml}$ ). In case of poor hormonal control, testicular ultrasonography can be considered even before the onset of puberty. Our observations suggest that tumour decrease can be obtained by aiming at adrenal oversuppression, but this usually requires high glucocorticoid doses and induces side effects. With asymptomatic tumours in young male patients with $\mathrm{CAH}$, a practical guideline could be to optimise adrenal suppression to a maximal tolerable glucocorticoid dose and to offer analysis and cryopreservation of semen as soon as the patient can be motivated.

\section{REFERENCES}

1. White PC, Speiser PW. Congenital adrenal hyperplasia due to 21-hydroxylase deficiency. Endocr Rev 2000; 21: 245-291.

2. Avila NA, Premkumar A, Shawker TH, Jones JV, Laue L, Cutler GB Jr. Testicular adrenal rest tissue in congenital adrenal hyperplasia: findings at gray-scale and color Doppler US. Radiology 1996; 198: 99-104.

3. Cabrera MS, Vogiatzi MG, New MI. Long term outcome in adult males with classic congenital adrenal hyperplasia. J Clin Endocrinol Metab 2001; 86: 30703078.

4. Stikkelbroeck NMML, Otten BJ, Pasic A, Jager GJ, Sweep CGJ, Noordam K, Hermus ARMM. High prevalence of testicular adrenal rest tumors, impaired spermatogenesis, and Leydig cell failure in adolescent and adult males with congenital adrenal hyperplasia. J Clin Endocrinol Metab 2001; 86: 5721-5728.

5. Merke DP. Bornstein SR, Avila NA, Chrousos GP. NIH conference. Future directions in the study and management of congenital adrenal hyperplasia due to 21-hydroxylase deficiency. Ann Intern Med 2002; 136: 320-334.

6. Rutgers JL, Young RH, Scully RE. The testicular tumor of the adrenogenital syndrome. A report of six cases and review of the literature on testicular masses in patients with adrenocortical disorders. Am J Surg Pathol 1988; 12: 503-513.

7. Walker BR, Skoog SJ, Winslow BH, Canning DA, Tank ES. Testis sparing surgery for steroid unresponsive testicular tumors of the adrenogenital syndrome. J Urol 1997; 157: 1460-1463.

8. WHO Laboratory Manual for the examination of human semen and sperm-cervical mucus interaction. Geneva: World Health Organization, 1999.

9. Kemink SAG, Frijns JTM, Hermus ARMM, Pieters GFFM, Smals AGH, Lichtenbelt WDV. Body composition determined by six different methods in women bilaterally adrenalectomized for treatment of Cushing's disease. J Clin Endocrinol Metab 1999; 84: 3991-3999.

10. Therasse $P$, Arbuck SG, Eisenhauer EA, Wanders J, Kaplan RS, Rubinstein L, Verweij J, Van Glabbeke M, van Oosterom AT, Christian MC, Gwyther SG. New guidelines to evaluate the response to treatment in solid tumors. European Organization for Research and Treatment of Cancer, National Cancer Institute of the United States, National Cancer Institute of Canada. J Natl Cancer Inst 2000; 92: 205-216.

11. Swinkels LM, van Hoof HJ, Ross HA, Smals AG, Benraad TJ. Concentrations of salivary testosterone and plasma total, non-sex-hormone-binding globulin-bound, and free testosterone in normal and hirsute women during administration of dexamethasone/synthetic corticotropin [see Comments]. Clin Chem 1991; 37: 180185.

12. Groschl M, Rauh M, Dorr HG. Cortisol and 17hydroxyprogesterone kinetics in saliva after oral administration of hydrocortisone in children and young adolescents with congenital adrenal hyperplasia due to 21-hydroxylase deficiency. J Clin Endocrinol Metab 2002; 87: 1200-1204.

13. Otten BJ, Wellen JJ, Rijken JC, Stoelinga GB, Benraad TJ. Salivary and plasma androstenedione and 17hydroxyprogesterone levels in congenital adrenal hyperplasia. J Clin Endocrinol Metab 1983; 57: 11501154.

14. Young MC, Cook N, Read GF, Hughes IA. The pharmacokinetics of low-dose dexamethasone in congenital adrenal hyperplasia. Eur J Clin Pharmacol 1989; 37: 75-77.

15. Blumberg-Tick J, Boudou P, Nahoul K, Schaison G. Testicular tumors in congenital adrenal hyperplasia: steroid measurements from adrenal and spermatic veins. J Clin Endocrinol Metab 1991; 73: 1129-1133.

16. Combes-Moukhovsky ME, Kottler ML, Valensi $P$, Boudou P, Sibony M, Attali JR. Gonadal and adrenal catheterization during adrenal suppression and gonadal stimulation in a patient with bilateral testicular tumors and congenital adrenal hyperplasia. J Clin Endocrinol Metab 1994; 79: 1390-1394.

17. Benvenga S, Smedile G, Lo GF, Trimarchi F. Testicular adrenal rests: evidence for luteinizing hormone receptors and for distinct types of testicular nodules differing 
for their autonomization. Eur J Endocrinol 1999; 141:

231-237.

18. Jaaskelainen J, Tiitinen A, Voutilainen R. Sexual function and fertility in adult females and males with congenital adrenal hyperplasia. Horm Res 2001; 56: 73-

80.

VOLUME 17, NO. 4, 2004 
Brought to you by | Radboud University Nijmegen (Radboud University Nijmegen) Authenticated | 172.16.1.226

Download Date | 7/13/12 9:19 AM 